\title{
Enhancing Fundraising with Refund Bonuses
}

\author{
Timothy N. Cason ${ }^{\mathrm{a}, *}$, Robertas Zubrickas ${ }^{\mathrm{b}}$ \\ ${ }^{a}$ Krannert School of Management, Purdue University, West Lafayette, IN 47907-2056, USA \\ ${ }^{b}$ Department of Economics, University of Bath, Bath BA2 7AY, UK
}

\begin{abstract}
The provision point mechanism as a method of funding threshold public goods is extended with refund bonuses. Each contributor not only has his contribution refunded in the case of insufficient contributions but also receives a refund bonus proportional to his proposed contribution. As long as the refund bonus pool does not exceed the net value of the public good, in equilibrium the public good is always provided and refund bonuses are never distributed. In this paper, we empirically investigate this extension of the provision point mechanism in a laboratory experiment by testing its properties on allocative and distributive efficiency, equilibrium coordination, and invariance to information distribution. Individuals respond to the incentives induced by refund bonuses as predicted, but systematic deviations exist that are consistent with quantal response equilibrium. Since this simple mechanism has considerable practical potential especially in crowdfunding, these promising initial results call for further experimental work.

Keywords: Public goods, provision point mechanism, refund bonus, crowdfunding JEL: C72, C92, D82, H41
\end{abstract}

\section{Introduction}

The voluntary provision of public goods remains one of the most challenging problems of economic design. Starting with the seminal works of Clarke (1971), Groves (1973), and Groves and Ledyard (1977), many novel solutions have been proposed to this problem, which at least until Olson (1965) was deemed impossible to resolve. Yet

\footnotetext{
${ }^{*}$ Corresponding author

Email addresses: cason@purdue.edu (Timothy N. Cason), r.zubrickas@bath.ac.uk (Robertas Zubrickas)
} 
when technological developments made the application of decentralized provision methods feasible, the choice of practitioners - particularly in crowdfunding - fell on the simple voluntary contribution mechanism or, with a restriction to threshold public goods made henceforth, the provision point mechanism of the same form used by Benjamin Franklin in 18th-century America. ${ }^{1}$ This choice was motivated by the simple structure of the mechanism and not its incentive properties, since it is fraught with the classical free riding problem and subject to the asymptotic non-provision result of Mailath and Postlewaite (1990).

Despite its weak implementation properties, the provision point mechanism helped raise over 5 billion US dollars (estimated) in crowdfunding revenue worldwide in 2013, a nearly ten-fold increase in revenue since 2009 (Massolution (2014)). ${ }^{2}$ However, a valid concern can be raised about the robustness of the present growth of crowdfunding, especially for community, charity, or creative projects. The current growth may be primarily driven through contributions submitted by the population of socially minded consumers, and growth may wane as this population is exhausted. ${ }^{3}$ Consequently, the problem of attracting contributions from a broader range of consumers will come to the fore if high levels of voluntary contributions are to be sustained and grown. As a possible solution to this problem, Zubrickas (2014) proposes a refinement to the provision point mechanism that improves its properties up to strict implementation while retaining its simple structure. The present paper is an experimental study of the proposed refinement and its properties.

The idea of the refinement is to introduce refund bonuses that are paid in the event of insufficient contributions. ${ }^{4}$ As Zubrickas $(2013,2014)$ demonstrates, with refund bonuses

\footnotetext{
${ }^{1}$ In the economics literature, the provision point mechanism was formally introduced by Palfrey and Rosenthal (1984) and Bagnoli and Lipman (1989). For reviews of experimental evidence on its performance, see Ledyard (1995) and Chen (2008).

${ }^{2}$ While Kickstarter, the largest crowdfunding platform, reports the success rate of $39 \%$ of its initially pre-screened fundraising campaigns (Kickstarter (2015)), the success rate can be as low as $10 \%$ for Indiegogo, the second largest (The Verge (2013)).

${ }^{3}$ For instance, an empirical study on crowdfunding Kuppuswamy and Bayus (2014) report that a campaigner's own social network and contributions coming from it make an important factor for the success of the project. With increasing project sizes, the role of this factor may accordingly diminish making it harder to reach the contribution target.

${ }^{4}$ Also see Tabarrok (1998) for a related idea.
} 
set to increase in own contribution, the only equilibrium outcome is the provision of public goods. Either competition for refund bonuses or preference for the public good induces sufficient contributions. For example, consider the problem of raising funds for a $\$ 10,000$ public project. As under the standard provision point mechanism, the mechanism designer solicits contributions toward the project, which is financed out of contributions if they are sufficient for this purpose. If the threshold is not reached, contributions are refunded and, additionally, the mechanism designer gives every contributor a refund bonus equal to, say, $10 \%$ of their contribution. ${ }^{5}$ As long as the net utility from the project exceeds the maximal amount of refund bonuses payable (i.e., $10 \%$ of $\$ 10,000$ ), the only equilibrium outcome is the implementation of the project and the refund bonuses are not dispensed. Furthermore, by manipulating refund bonus rules it is possible to implement the project in a unique equilibrium with Lindahl prices. Lastly, on the normative side of analysis, the mechanism ensures that an individually rational contributor always gets a positive utility either from the public good or from the refund bonus whereas free-riders may end up with nothing.

In the present paper, we test the equilibrium predictions of this new provision point mechanism brought about by the introduction of refund bonuses. We form hypotheses related to allocative efficiency, equilibrium coordination, distributive efficiency, and invariance to information distribution. The latter hypothesis draws on the aggregative structure of the mechanism that implies that only the knowledge of aggregates matters for individual contribution behavior. Our experimental results offer empirical support for Nash equilibrium predictions by demonstrating that consumers respond to economic incentives in predicted ways. The most distinguishing prediction is the non-monotonous rate of provision with respect to the level of refund bonus. Namely, too generous refund bonuses will work against provision as consumers would rather coordinate on a total contribution just short of the provision point. Our empirical results exactly demonstrate this relationship: Despite generous refund bonuses, consumers choose to contribute less but,

\footnotetext{
${ }^{5}$ The assumption is that the mechanism designer has a budget of her own, but one could think of many alternative sources of refund bonuses, e.g., seed money initially raised from first movers.
} 
in line with the theoretical prediction, only marginally less as we observe little difference in total contributions across different refund bonus treatments. We also compare the provision point mechanisms with and without refund bonuses. We find expected differences in performance levels in favor of refund bonuses, which occur with a larger group size and heterogeneous population. With respect to predictions on invariance to information distribution and, in part, on equilibrium coordination, the closest fit is obtained when we refine the theoretical model with bounded rationality that underlies the quantal response equilibrium (Rosenthal (1989), McKelvey and Palfrey (1995)).

The present paper belongs to the strand of literature on non-coercive methods of fundraising for public projects. The idea of using pecuniary incentives in order to induce contributions appears in a number of works. For example, Falkinger (1996) studies a mechanism that rewards contributors with above-average contributions, and Morgan (2000) studies one that induces contributions with the help of lotteries. Goeree et al. (2005) demonstrate the advantages of the all-pay auction design in fundraising. Gerber and Wichardt (2009) study a multi-stage mechanism that pre-commits consumers to optimal contributions with conditionally refundable deposits. For experimental evidence on the performance of these mechanisms, see Falkinger et al. (2000), Morgan and Sefton (2000), Lange et al. (2007), Corazzini et al. (2010), and Gerber et al. (2013) who all report improved allocative efficiency. For alternative fundraising methods, also see Varian (1994), Kominers and Weyl (2012), and most recently Masuda et al. (2014). However, apart from the lottery mechanism, the practical applicability of these mechanisms is questionable because of their complexity and concerns for group manipulability (which does not arise in our mechanism with refund bonuses). Furthermore, in many of these mechanisms distributive efficiency is likely to fail as, e.g., Kearny (2005) empirically demonstrates in the case of the lottery mechanism which proves highly regressive.

The remainder of the paper is organized as follows. Section 2 presents the provision point mechanism with refund bonuses, discusses its properties, and formalizes testable hypotheses. Section 3 describes the experimental design and Section 4 presents the empirical results. In Section 5, we refine the model with bounded rationality and discuss 
empirical implications. The last section concludes.

\section{Refund bonuses and theoretical predictions}

\subsection{Model}

There is a public good $(\mathrm{PG})$ project that benefits a group of $N$ consumers. The cost of the project is $C$ and its total value to the consumers is $V$, with $V>C$. Consumer $i$ 's individual value for the $\mathrm{PG}$ is given by $v_{i}, i=1, \ldots, N$, where $v_{i} \in[0, C)$ and $\sum_{i} v_{i}=V$. We assume that there is no aggregate uncertainty about the value of the project, namely, the total value of the project $V$ is public information, whereas individual values are private information. ${ }^{6}$

A mechanism designer solicits voluntary contributions toward the project. Let $g_{i}$ denote consumer $i$ 's contribution and $G$ the sum of contributions. If $G \geq C$, the project is funded out of the contributions collected, with the excess amount $G-C$ wasted (assumed for the ease of exposition). If $G<C$, the project is not implemented and each contributor gets his contribution refunded and, in addition, a refund bonus as a percentage $r$ of his own contribution. The refund bonus rule $r>0$, also referred to as simply the refund bonus $r$, is announced by the mechanism designer in the beginning of the campaign. We assume that the designer possesses a budget out of which she pays refund bonuses in the case of insufficient contributions. We denote the maximal amount of refund bonuses payable in the limit by $R=r C$.

Under the proposed scheme, the payoff to consumer $i$ is given by

$$
\pi_{i}\left(g_{i}, G\right)=\mathbb{1}(G \geq C)\left[v_{i}-g_{i}\right]+\mathbb{1}(G<C)\left[r g_{i}\right]
$$

where $\mathbb{1}($.$) is an index function. We assume that consumers choose contributions (without$ randomizing) that constitute a Nash equilibrium of the game induced by mechanism $r$,

\footnotetext{
${ }^{6}$ Such an information structure can arise when the group is a representative sample, up to the first moment, randomly drawn from the population of consumers with a known distribution of individual values. As a practical example, if valuations for street refurbishment depend on cars driven, then a fair estimate of the average valuation is arguably public knowledge. A similar incomplete information - known sum environment is considered in the experimental study of the provision point mechanism by Marks and Croson (1999), among others.
} 
which is short for the mechanism with refund bonus share $r$. Letting $G_{-i}$ denote the sum of all contributions of consumers other than $i$, we define

Definition 1. A vector of contributions $\mathbf{g}^{*}=\left(g_{1}^{*}, \ldots, g_{N}^{*}\right)$ is a Nash equilibrium if $g_{i}^{*}$ maximizes $\pi_{i}\left(g_{i}, G_{-i}^{*}+g_{i}\right)$ for each $i$.

The next proposition characterizes the set of pure-strategy Nash equilibria, which we denote by $\Gamma(r)$.

Proposition 1. If $0<R \leq V-C$, then $\Gamma(r)=\left\{\mathbf{g}^{*}: \forall i, g_{i}^{*} \leq \frac{1}{1+r} v_{i}, G^{*}=C\right\}$. Otherwise, $\Gamma(r)=\{\emptyset\}$.

Proof. In equilibrium, $G^{*}<C$ cannot hold as any consumer could obtain a higher refund bonus by marginally increasing his contribution because of $r>0$. Likewise, any consumer with a positive contribution could gain in utility by marginally decreasing his contribution if $G^{*}>C$. Thus, the equilibrium candidates need to have $G^{*}=C$. A vector $\mathrm{g}^{*}$ is an equilibrium if for each consumer $i$ the net utility from the PG, $v_{i}-g_{i}^{*}$, exceeds the highest possible refund bonus, $r g_{i}^{*}$, or after transformations

$$
g_{i}^{*} \leq \frac{1}{1+r} v_{i}
$$

Summing up (2) yields $V-C \geq R$, which is the condition for equilibrium existence as it ensures that there is a vector $\mathbf{g}^{*}$ such that (2) is satisfied for all $i$. If $R>V-C$, then $G^{*}=C$ cannot hold as (2) will be violated at least for some $i$, implying $\Gamma(r)=\{\emptyset\}$.

\subsection{Equilibrium properties}

According to Proposition 1, the introduction of refund bonuses induces sufficient contributions provided that refund bonuses are not too generous. Intuitively, with a refund amount that is increasing in own contribution the mechanism provides incentives for consumers to contribute. Whether the consumers reach the target for provision or contribute an amount just short of the target depends on what brings greater utility, the refund bonuses or the PG. If the net utility of the public good, $V-C$, exceeds the maximal amount of refund bonuses $R$, then they seek to reach the target, and vice versa. 
Another important property of the mechanism is that an increase in refund bonus $r$ reduces the set of equilibria, which formally follows from condition $(2)-$ if $r^{\prime}>r$, then $\Gamma\left(r^{\prime}\right) \subseteq \Gamma(r)$. Intuitively, as refund bonuses make deviations profitable, in equilibrium each consumer has to obtain a net utility level from the PG at least as high as that from the refund bonus if he deviates. A larger refund bonus implies a more profitable deviation, which, in turn, implies a higher level of utility for each consumer in equilibrium. As a result, fewer contribution profiles can be sustained as equilibria. ${ }^{7}$ In fact, when the maximal amount of refund bonuses is set at the net value of the public good, $R=$ $V-C$, the mechanism implements the public good uniquely, altogether overcoming the equilibrium coordination problem. Furthermore, the unique equilibrium has a special feature. All consumers contribute the same proportion of their values, i.e., $g_{i}=\frac{C}{V} v_{i}$, resulting in distributive efficiency.

The game induced by the mechanism is aggregative and, furthermore, aggregate invariant - the sum of individual payoffs, $\sum_{i} \pi_{i}$, depends on aggregates $V$ and $G$ only and is independent of the distribution of individual contributions or valuations. Put differently, what matters for individual decisions is the aggregate valuation of the PG, not the distribution of individual valuations and not even the knowledge of valuations of other consumers. Thus, as long as individual valuations sum to the same amount, their distribution does not affect the equilibrium outcome.

We illustrate the mechanism and its equilibrium properties with an example, which introduces the experimental parameterization and forms the basis for our experimental hypotheses. Suppose that the PG project costs $C=200$, for which a group of $N=5$ consumers has an aggregate valuation of $V=\sum_{i=1}^{5} v_{i}=300$. When the refund bonus is set at $r=0.25$, the equilibrium set is $\Gamma(0.25)=\left\{\mathbf{g}^{*}: \forall i, g_{i}^{*} \leq \frac{4}{5} v_{i}, G^{*}=200\right\}$, and when it is increased to $r=0.50$, the equilibrium set becomes singleton, $\Gamma(0.50)=$ $\left\{\mathbf{g}^{*}: \forall i, g_{i}^{*}=\frac{2}{3} v_{i}, G^{*}=200\right\}$. However, when the refund bonus is set at $r=0.75$, there is no equilibrium in continuous strategies. With discrete strategies, the equilibrium set is

\footnotetext{
${ }^{7}$ This also implies that certain normative relative contribution rules are no longer equilibria as $r$ rises, such as equality of earnings or equality of contributions (Reuben and Riedl (2013)).
} 


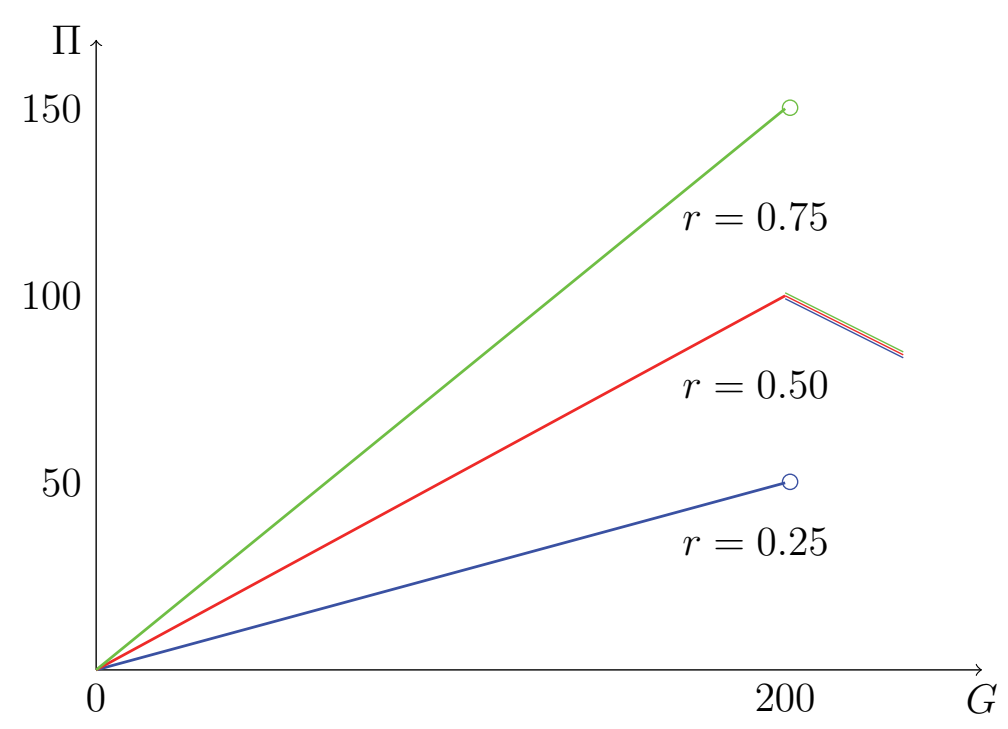

Figure 1: Total Payoff $\Pi(G)$

found to be $\Gamma(0.75)=\left\{\mathbf{g}^{*}: \forall i, g_{i}^{*} \geq \frac{4}{7} v_{i}, G^{*}=200-\epsilon\right\}$, where $\epsilon$ is the smallest currency unit.

Figure 1 plots the total payoffs, which we denote by $\Pi(G)$, for these three different refund bonus levels. The refund bonus tilts upwards the total payoff graph on the interval of aggregate contributions below the provision point. At refund bonus $r=0.25$, the payoff function $\Pi(G)$ is discontinuous at $G=200$ yet upper semicontinuous, at $r=0.50$ it is continuous, which explains the equilibrium uniqueness, but at $r=0.75$ the upper semicontinuity is no longer preserved, which is behind the non-existence of equilibria in continuous strategies.

Finally, the equilibrium outcome of Proposition 1 can also be achieved with mechanisms using different refund bonus schemes. The most important condition is that the refund bonus amount increases in own contribution. For instance, consider the mechanism where in the case of insufficient contributions the designer promises to distribute a fixed amount of bonus money $M$ among the contributors in shares $\frac{g_{i}}{G} M$. It is straightforward to demonstrate that this mechanism is equivalent in equilibrium outcomes, both at individual and aggregate levels, to the analyzed mechanism $r$ where $r=M / C$. In our example, mechanisms $r=0.25,0.50$, and 0.75 are equivalent to mechanisms with bonus money $M=50,100$, and 150 , respectively. 


\subsection{Hypotheses}

For the PG project with parameter values $V=300, C=200$, and the smallest currency unit $\epsilon=1$ we articulate five hypotheses that deal with the equilibrium predictions of the mechanisms $r=0.25,0.50$, and 0.75 .

Hypothesis 1 (Allocation). The rate of $P G$ provision is similar for refund bonus $r=$ 0.25 and $r=0.50$, but is lower for $r=0.75$.

Hypothesis 2 (Aggregate contributions). Aggregate contributions are similar for all refund bonus shares $r$.

Hypothesis 3 (Coordination). For $r=0.25,0.50$, aggregate contribution tends toward 200 but for $r=0.75$ toward 199. The equilibrium is reached faster for $r=0.50$.

Hypothesis 4 (Information/Value asymmetry). Aggregate outcomes are independent of the distribution of individual values.

Hypothesis 5 (Distributive efficiency). For the mechanism $r=0.50$, contributions tend toward two thirds of individual values.

Lastly, we also make an attempt to compare performance of mechanisms with and without refund bonuses. In our example, under the standard provision point mechanism $(r=0)$ the set of equilibria consists of the subset of inefficient equilibria, $\left\{\mathbf{g}^{*}: G^{*} \leq\right.$ $\left.200-\max \left(v_{i}\right)\right\}$, and the subset of efficient equilibria, $\left\{\mathbf{g}^{*}: \forall i, g_{i}^{*} \leq v_{i}, G^{*}=200\right\}$. Regarding equilibrium selection, two opposing predictions are distinguished in the economics literature. On one hand, Bagnoli and Lipman (1989) argue that inefficient equilibria are not robust to the refinement of undominated perfect equilibrium. Intuitively, if consumers' actions are subject to trembles, then a positive contribution dominates a zero contribution. $^{8}$ On the other hand, Mailath and Postlewaite (1990) argue that the inefficient outcome is more likely to emerge if the size of population is large. In particular, if a consumer's contribution is pivotal only with a small probability, then the consumer is better off by contributing nothing. Thus, we hypothesize

${ }^{8}$ This argument does not hold, however, in the presence of nuisance costs of contribution. See Castillo et al. (2014) on the salience of nuisance costs for Internet donations. 
Hypothesis 6 (No refund bonuses). The rate of provision of the mechanism without refund bonuses is similar to that of the mechanism with refund bonuses when group size is small but is lower when group size becomes larger.

\section{Experimental Design and Procedures}

Design

We report data gathered from a total of 530 human subjects. The main experiment features a 4x2 design, with 3 levels of the refund bonus $(r=0.25,0.50$ and 0.75$)$ or no refund bonus $(r=0)$, and heterogeneous or homogeneous values for the public good. Subjects were placed in fixed, 5-person groups, and the threshold to fund a "group project" was always fixed at $G=200$ experimental dollars. In the homogeneous values treatment each individual earned a payment of $v=60$ if the project was funded. In the heterogeneous values treatment each individual received a payment drawn randomly for the round without replacement from $\{40,50,60,70,80\}$. Subjects learned their drawn $v$ before making their group project allocation decision each period.

The heterogeneous versus homogeneous value treatment was varied across subjects; that is, either all periods of a session employed heterogeneous values or all periods of a session employed homogeneous values. As shown in Table 1, the refund bonus was varied within and across subjects. The first 30 periods of each session employed one value of $r$ and the second 30 periods of each session employed another value of $r$. The sequence was varied in every possible combination to control for order effects. With the exception of the no bonus condition for the late periods 31-60, at least 9 different groups of 5 participated in each refund bonus condition for the two values environments.

Most sessions were conducted with 25 subjects (5 groups), although a few sessions were smaller due to subject no-shows. The 5-person groups remained fixed for all 60 periods. This allows us to treat each group as an independent observation, and should also promote learning in this coordination game with multiple equilibria. The final period was not announced in advance.

We supplemented the main experimental design summarized in Table 1 with 90 additional subjects in two follow-up environments all with the homogeneous $(v=60$ for all 
Table 1: Main Experimental Design

\begin{tabular}{ccccc}
\hline \hline \multirow{2}{*}{ Payment $v$} & \multicolumn{2}{c}{ Refund bonus } & Number of & Number of \\
& Periods 1-30 & Periods 31-60 & Subjects & Groups \\
\hline 60 & $r=0.25$ & $r=0.50$ & 25 & 5 \\
60 & $r=0.25$ & $r=0.75$ & 25 & 5 \\
60 & $r=0.25$ & $r=0$ & 25 & 5 \\
60 & $r=0.50$ & $r=0.25$ & 25 & 5 \\
60 & $r=0.50$ & $r=0.75$ & 20 & 4 \\
60 & $r=0.75$ & $r=0.25$ & 25 & 5 \\
60 & $r=0.75$ & $r=0.50$ & 25 & 5 \\
60 & $r=0$ & $r=0.50$ & 20 & 4 \\
60 & $r=0$ & $r=0.75$ & 25 & 5 \\
\hline$\{40,50,60,70,80\}$ & $r=0.25$ & $r=0.50$ & 25 & 5 \\
$\{40,50,60,70,80\}$ & $r=0.25$ & $r=0.75$ & 25 & 5 \\
$\{40,50,60,70,80\}$ & $r=0.50$ & $r=0.25$ & 25 & 5 \\
$\{40,50,60,70,80\}$ & $r=0.50$ & $r=0.75$ & 25 & 5 \\
$\{40,50,60,70,80\}$ & $r=0.50$ & $r=0$ & 25 & 5 \\
$\{40,50,60,70,80\}$ & $r=0.75$ & $r=0.25$ & 25 & 5 \\
$\{40,50,60,70,80\}$ & $r=0.75$ & $r=0.50$ & 25 & 5 \\
$\{40,50,60,70,80\}$ & $r=0$ & $r=0.25$ & 25 & 5 \\
$\{40,50,60,70,80\}$ & $r=0$ & $r=0.75$ & 25 & 5 \\
\hline \hline
\end{tabular}

consumers) value condition. As in the main experiment, these follow-up sessions included 60 periods with a treatment switch after period 30. One follow-up environment increased the group size to $N=10$ in order to evaluate Hypothesis 6. When making this change in group size we simultaneously increase the threshold to $G=400$ to fund the public good to keep the "step return" constant at 1.5 (Croson and Marks (2000)). This is similar to the group size change in Bagnoli and McKee (1991). A total of 40 subjects (4 fixed and independent groups) participated in these larger group sessions, 20 beginning with no bonus and switching to a refund bonus of 0.25 and 20 beginning with a refund bonus of 0.25 and switching to no bonus.

The second follow-up environment changed the refund bonus from a fraction of the consumers' contribution to a share of a fixed fund that is proportional to the amount of her contribution relative to the total contributions made by members of her group. As noted above, this version of the mechanism has identical incentive properties and the same equilibria as the more simplified refund bonus version implemented in the main 
experiment. A total of 50 subjects (10 fixed and independent groups) participated in these sessions, 25 beginning with a fund of 50 (equivalent to the 50/200 $=0.25$ refund bonus) and switching to a fund of 150 (equivalent to the 150/200 $=0.75$ refund bonus), and 25 beginning with a fund of 150 and switching to a fund of 50 . This version of the mechanism performs similarly to the refund fraction version, so we do not report the results explicitly.

\section{Procedures}

An experimenter reads the instructions (distributed to subjects in hardcopy and shown in the Appendix) at the beginning of the session to establish common knowledge of the game and decision task among participants. Following this instruction phase all interactions were handled anonymously using a computer program written in zTree (Fischbacher (2007)). At the end of each decision period subjects learned the total amount allocated to the "group project," whether the project reached the provision point threshold and was funded, the subject's own refund bonus if the project was not funded, as well as period and cumulative experimental dollar earnings. Subjects were required to record this information on a hardcopy record sheet to emphasize the saliency of this information and to provide easy access to the game history.

Earnings accumulated across all 60 periods and were converted at a rate of 50 experimental dollars $=1$ U.S. dollar and paid in cash immediately at the conclusion of each session. Each subject also began their session with an initial endowment of 200 experimental dollars. Sessions typically lasted about 90 minutes and subjects earned an average of $\$ 25.25$ each. Subjects were recruited by email through a random draw from a subject database of approximately 3,000 of the 30,000 Purdue University undergraduate students using ORSEE (Greiner (2004)). All sessions were conducted at the Vernon Smith Experimental Economics Laboratory (VSEEL) at Purdue and no subject participated in more than one session. 


\section{Results}

We present the empirical findings in a series of numbered results that correspond to the numbered hypotheses, followed by their statistical support. The first section considers the mechanism with refund bonuses, and the second section compares the refund bonus mechanism to the provision problem with no refund bonuses. Unless noted otherwise, the reported statistical tests employ a five-percent significance threshold.

\subsection{Performance Across Refund Bonus Treatments}

Result 1 (Allocation). The public good is funded significantly less frequently when the refund bonus is set at the high level (0.75), compared to the 0.50 and 0.25 refund bonuses. This holds for both the homogeneous and heterogeneous value environments and becomes stronger over time. With heterogeneous values, PG provision is more frequent with the 0.25 than the 0.50 refund bonus. For the 0.50 refund bonus only, the PG is funded more frequently in the homogeneous than the heterogeneous value environment.

Support: Figure 2 reports the five-period moving average of the frequency that the PG is funded for each refund bonus treatment. Panel A displays results from the homogeneous value environment, and the declining trend in the 0.75 refund bonus treatment contrasts sharply with the stable or rising trend in the other two refund bonus treatments. The difference between the 0.75 treatment and the other two treatments is statistically significant in both the early 30 and the late 30 periods. For statistical tests we employ probit models with group-level random effects and a linear time trend. Identical conclusions follow from nonparametric Wilcoxon rank-sum tests, using independent groups as the unit of observation. ${ }^{9}$

Panel B displays results from the heterogeneous value environment, and the only noticeable difference from Panel $\mathrm{A}$ is the downward trend and lower level of the PG funding rate for the 0.50 treatment. The 0.25 refund bonus $\mathrm{PG}$ funding rate is significantly

\footnotetext{
${ }^{9}$ The data exhibit hysteresis, since the initial periods 31-40 following the treatment switchover appear to be influenced by the earlier treatment; for example, the PG is funded more (less) frequently in these periods if the earlier treatment was the high (low)-funding 0.25 (0.75) treatment. To minimize this nuisance variation and allow for some initial learning, the nonparametric tests summarized here exclude periods 1-10 and periods 31-40.
} 

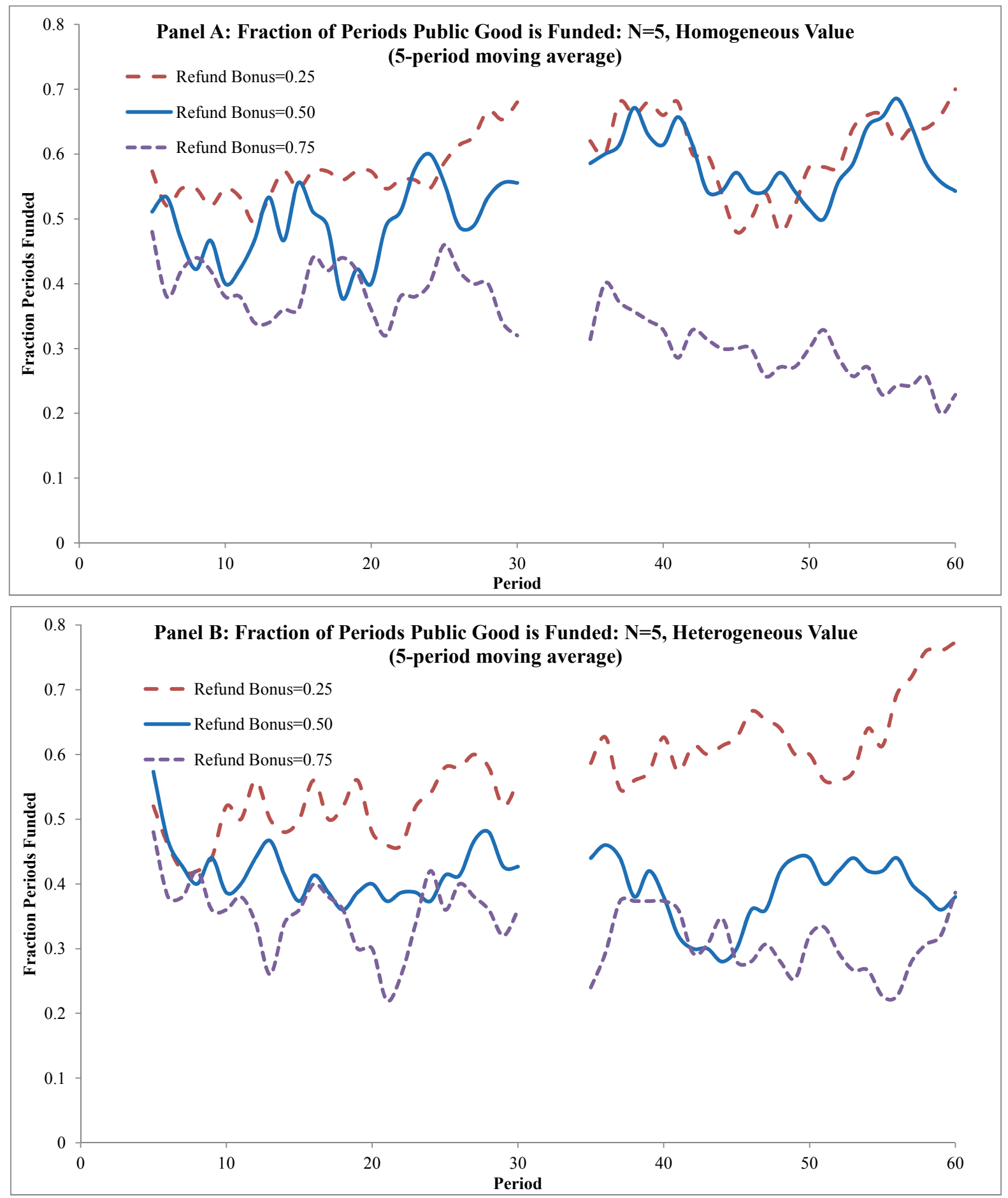

Figure 2: Fraction of Periods Public Good is Funded

greater than the rate for the 0.75 and 0.50 refund bonus in both the early 30 and the late 30 periods, and the rates for the 0.50 and 0.75 treatments are significantly different for the late 30 periods. When controlling for the refund bonus level, funding rates differ between the homogeneous and heterogeneous value environments only when the refund bonus $=0.50$. This holds for both the early and late 30 periods using nonparametric 
Wilcoxon tests and random effects probit regressions, although for the regressions the difference in the early 30 periods is significant at only the ten-percent level.

Based on the Nash equilibria for this mechanism, the significantly greater PG provision rate for the 0.25 treatment compared to the 0.50 treatment for the heterogeneous treatment is surprising. Although all equilibria are efficient in both of these treatments, in the sense that the public good is funded and contributions are not wasted, the equilibrium is unique in the 0.50 treatment. As noted earlier, we conjectured that this would promote equilibrium play, since it resolves the coordination problem that arises through the multiple equilibria of the 0.25 treatment. As we explain below in Section 5, however, the expected costs of equilibrium deviations are much larger in the 0.25 than the 0.50 treatment, so the QRE model of boundedly rational choice is consistent with the greater rate of efficient PG provision in the 0.25 refund bonus condition.

Result 2 (Aggregate Contributions). Total contributions to the PG are near the 200 threshold in all treatments, but they are sometimes significantly lower when the refund bonus is at the high level (0.75) and significantly higher when the refund bonus is at the low level (0.25). This divergence occurs more often in the later periods, and when values are heterogeneous. Total contributions are also lower in the heterogeneous than the homogeneous treatment, except when the refund bonus is 0.25.

Support: Figure 3 displays the five-period moving average of the average aggregate contributions for each refund bonus treatment. The averages are all between 195 and 201 in the late periods of the homogeneous value condition shown in Panel $\mathrm{A}$, and the 0.75 refund bonus is significantly below the 0.50 refund bonus treatment in periods 31-60. (Statistical tests are based on a random effects regression and confirmed with nonparametric Wilcoxon tests.) The differences in average contributions are greater in the heterogeneous value condition (Panel B), and the 0.25 refund bonus treatment is significantly greater than the other two refund bonus treatments in the late periods $31-60$ and the 0.75 refund bonus treatment in periods 1-30 as well. Average contributions are also significantly greater in the heterogeneous than the homogeneous condition when the refund bonus is 0.25 (for late periods 31-60); but they are significantly lower in the heterogeneous than 


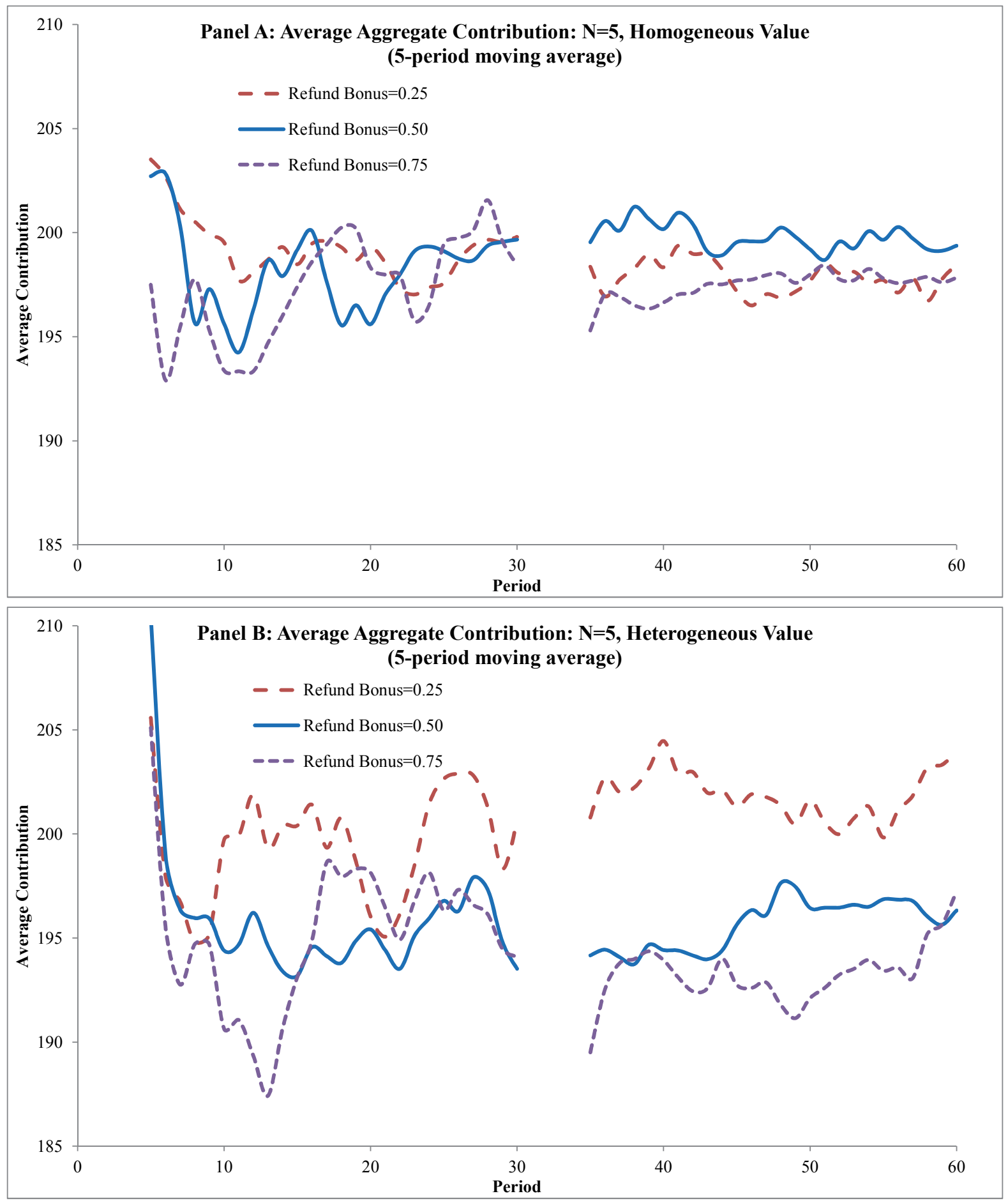

Figure 3: Average Aggregate Contribution

homogeneous condition when the refund bonus is 0.50 or $0.75 .^{10}$ Again, as we argue

\footnotetext{
${ }^{10}$ This conclusion holds for both the random effects regressions and the nonparametric Wilcoxon tests for the late periods, but for the early periods the differences between homogeneous and heterogeneous treatments for 0.50 and 0.75 are only significant using the Wilcoxon tests. As noted in the previous footnote, for the independent group-level averages employed in the nonparametric tests we exclude periods 1-10 and 31-40 to minimize the influence of initial learning and hysteresis effects.
} 
below, the observed deviation from the Nash equilibrium play is consistent with a model of bounded rationality.

Result 3 (Coordination). In the homogeneous values environment, groups coordinate to repeat aggregate contributions on exactly 200 in the 0.25 and 0.50 refund bonus treatments and on exactly 199 in the 0.75 refund bonus treatment and in later periods. The variance in neither aggregate nor individual contributions is significantly different across refund bonus conditions.

Table 2: Estimated Likelihood of Repeating Aggregate Contributions of 200 or 199 (Homogeneous Values Environment)

\begin{tabular}{|c|c|c|c|c|c|c|c|}
\hline & & \multicolumn{3}{|c|}{ Early periods $1-30$} & \multicolumn{3}{|c|}{ Late periods $1-30$} \\
\hline & & $\mathrm{r}=0.25$ & $\mathrm{r}=0.50$ & $\mathrm{r}=0.75$ & $\mathrm{r}=0.25$ & $\mathrm{r}=0.50$ & $\mathrm{r}=0.75$ \\
\hline \multicolumn{8}{|l|}{$\begin{array}{l}G=200 \text { in } \\
\text { period } t \text { if: }\end{array}$} \\
\hline & $\begin{array}{l}G=200 \text { in } \\
\text { period } t-1\end{array}$ & 0.438 & 0.500 & 0.154 & 0.732 & 0.698 & 0.134 \\
\hline & $\begin{array}{l}G \neq 200 \text { in } \\
\text { period } t-1\end{array}$ & 0.121 & 0.134 & 0.146 & 0.184 & 0.178 & 0.238 \\
\hline \multicolumn{8}{|l|}{$\begin{array}{l}G=199 \text { in } \\
\text { period } t \text { if: }\end{array}$} \\
\hline & $\begin{array}{l}G=199 \text { in } \\
\text { period } t-1\end{array}$ & 0.147 & 0.145 & 0.325 & 0.295 & 0.408 & 0.549 \\
\hline & $\begin{array}{l}G \neq 199 \text { in } \\
\text { period } t-1\end{array}$ & 0.063 & 0.064 & 0.060 & 0.144 & 0.136 & 0.111 \\
\hline
\end{tabular}

Note: Likelihoods estimated from a random effects probit model (marginal effects displayed).

Support: Table 2 reports the likelihood of groups repeating aggregate contributions of 200 or 199 in consecutive periods, displaying marginal effects of a series of probit regression models. The top section of the table indicates that groups contribute an aggregate amount of 200 relatively infrequently (12 to 24 percent of the time) if they did not contribute 200 in the previous period. If groups contributed 200 in the previous period and the refund bonus is 0.25 or 0.50 , then the likelihood they contribute 200 again triples or quadruples, which is in line with our prediction. This increase is highly statistically significant $(p-$ values $<0.01)$. By contrast, groups do not significantly change their likelihood of contributing 200 in this case when the refund bonus is 0.75 . 
The lower part of Table 2 shows that groups contribute an aggregate amount of 199 between 6 and 14 percent of the time if they did not contribute 199 in the previous period. The likelihood they contribute 199 is greater when they contribute 199 in the previous period, but in the early periods 1-30 this increase is not statistically significant when the refund bonus is 0.25 or 0.50 . The increase is much larger and is highly significant when the refund bonus is 0.75 and in the late periods 31-60. Similar probit models estimated for the heterogeneous values environment provide substantially weaker results because of the low frequency that groups exactly chose 200 or 199.

Since the Nash equilibrium is unique in only the 0.50 refund bonus treatment, we expected contribution variance to be lower in this treatment because the coordination problem of multiple equilibria is avoided. Individual contribution variances, however, are usually not significantly different across the refund bonus treatments. The contribution variance is lower in the 0.50 refund than the 0.25 refund bonus treatment only in the late periods for the heterogeneous values treatment. No other pairwise differences across refund bonus treatments are statistically significant. (These conclusions are identical both for random effects regressions and for nonparametric Wilcoxon tests.)

Result 4 (Information/Value Asymmetry). Variance in total contributions is greater with heterogeneous than with homogeneous valuations in all refund bonus conditions.

Table 3: Average Standard Deviation in Aggregate Group Contributions

\begin{tabular}{lcccccc}
\hline & \multicolumn{2}{c}{$r=0.25$} & \multicolumn{2}{c}{$r=0.50$} & \multicolumn{2}{c}{$r=0.75$} \\
\cline { 2 - 7 } & $\begin{array}{c}\text { Homog. } \\
\text { values }\end{array}$ & $\begin{array}{c}\text { Heterog. } \\
\text { values }\end{array}$ & $\begin{array}{c}\text { Homog. } \\
\text { values }\end{array}$ & $\begin{array}{c}\text { Heterog. } \\
\text { values }\end{array}$ & $\begin{array}{c}\text { Homog. } \\
\text { values }\end{array}$ & $\begin{array}{c}\text { Heterog. } \\
\text { values }\end{array}$ \\
\hline Periods 11-30 & 7.3 & 14.6 & 9.5 & 14.7 & 9.0 & 15.0 \\
Periods 41-60 & 4.9 & 10.9 & 4.1 & 10.3 & 3.4 & 11.4 \\
\hline \hline
\end{tabular}

Support: Table 3 displays the standard deviation in group contributions for the early and late periods, averaged across the independent groups in each treatment. (As in the previous analyses, we exclude periods 1-10 and 31-40 to minimize the influence of initial learning and hysteresis effects.) In contrast to our prediction, the variance of total group contributions is significantly greater in the heterogeneous than the homogeneous 
value environments in both the early and late periods for the 0.25 and 0.75 refund bonus treatments and in the late periods of the 0.50 refund bonus treatment. This table also illustrates the decline in contribution variance over time, indicating greater coordination.

Result 5 (Distributive Efficiency). Individual contributions to the $P G$ increase in the consumer's PG value in the heterogeneous value environment, resulting in (approximate) distributive efficiency. This holds across all refund bonus conditions.

Support: Figure 4 displays scatterplots of individual contributions for each PG value in the heterogeneous values environment for the later periods 41-60. (We omit the similar figures for the early periods, which-as already documented-exhibit considerably greater variance.) While clearly the subjects do not contribute a constant fraction of their value, the displayed linear contribution function explains about half or more of the variance in contributions in these later periods. Moreover, the fitted contribution function is similar to the $2 / 3$ value that results in distributive efficiency. The fitted regression lines are estimated with considerable precision, however, and can reject the joint hypothesis that the contribution $=2 / 3$ value in all three refund bonus treatments.

\subsection{Comparison to Provision without Refund Bonuses}

The analysis to this point considers only the three treatments with refund bonuses. Consistent with theory, results show that provision is more common in the 0.25 and 0.50 refund bonus treatments in which non-provision is not an equilibrium, compared to the 0.75 refund bonus treatment. Provision and contributions are greatest for the 0.25 refund bonus treatment. In this section we compare provision rates and contributions of this treatment with the provision point problem without refund bonuses.

Result 6 (No Refund Bonuses). Without refund bonuses, the public good is funded significantly less frequently in late periods with heterogeneous values and with larger (10 person) groups compared to the 0.25 refund bonus. Average group contributions are also sometimes significantly lower without refund bonuses.

Support: Figure 5A displays the rate that the PG is funded for the treatment without refund bonuses, compared to the treatment with a refund bonus of 0.25 , and Figure $5 \mathrm{~B}$ 

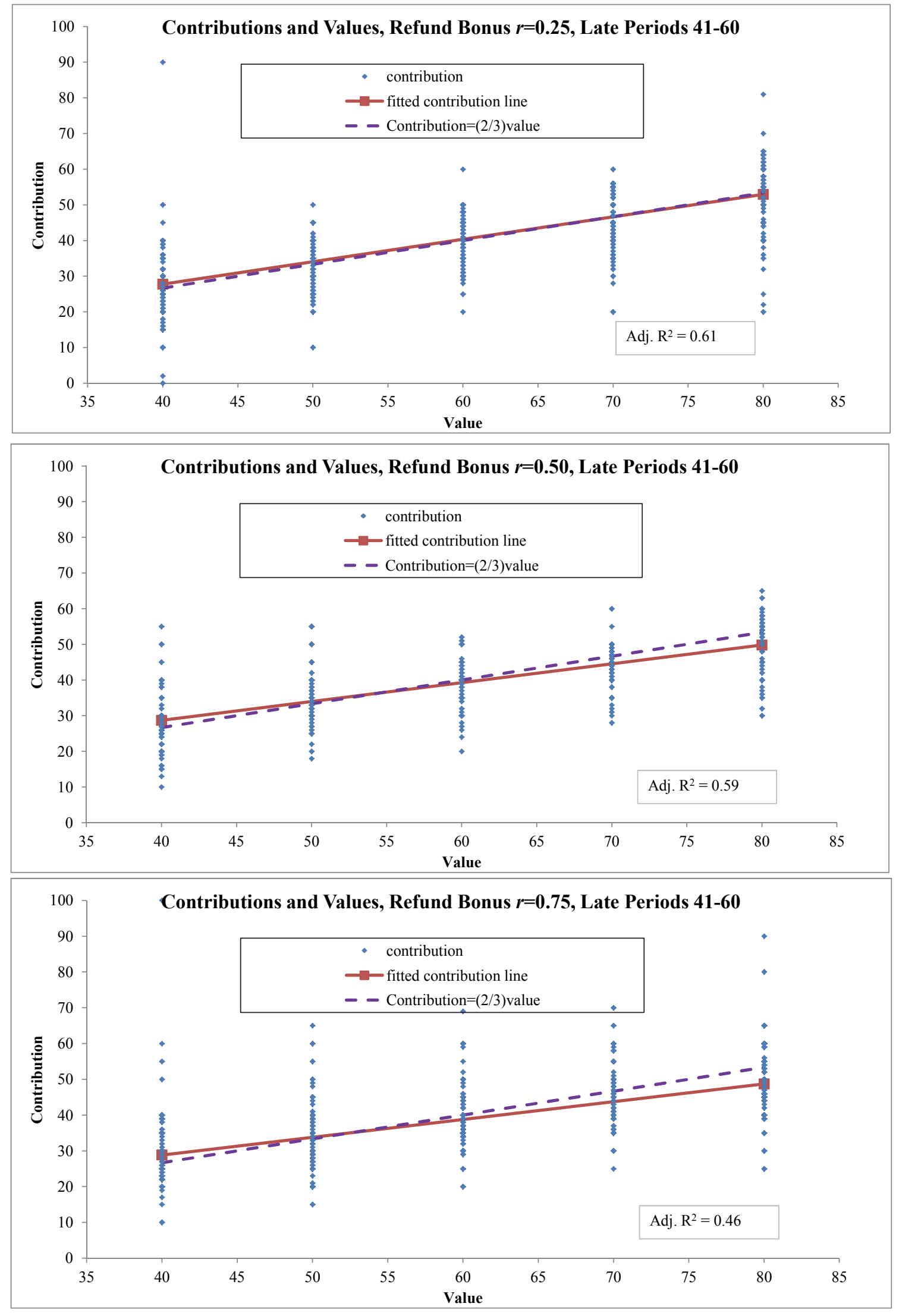

Figure 4: Contributions and Values 
provides a similar comparisons for average individual contributions. The experimental design includes data for both homogeneous and heterogeneous value conditions for the small groups $(N=5)$, but only homogeneous values for the large groups $(N=10)$. Funding rates and average contributions without refund bonuses are usually lower than or similar to the 0.25 refund bonus mechanism, with the exception of the late periods in the homogeneous $N=5$ treatment. (Only 25 subjects participated in the late periods for this treatment, and the performance in this treatment is not significantly different from the 0.25 refund bonus treatment.) For statistical comparisons we employ the same
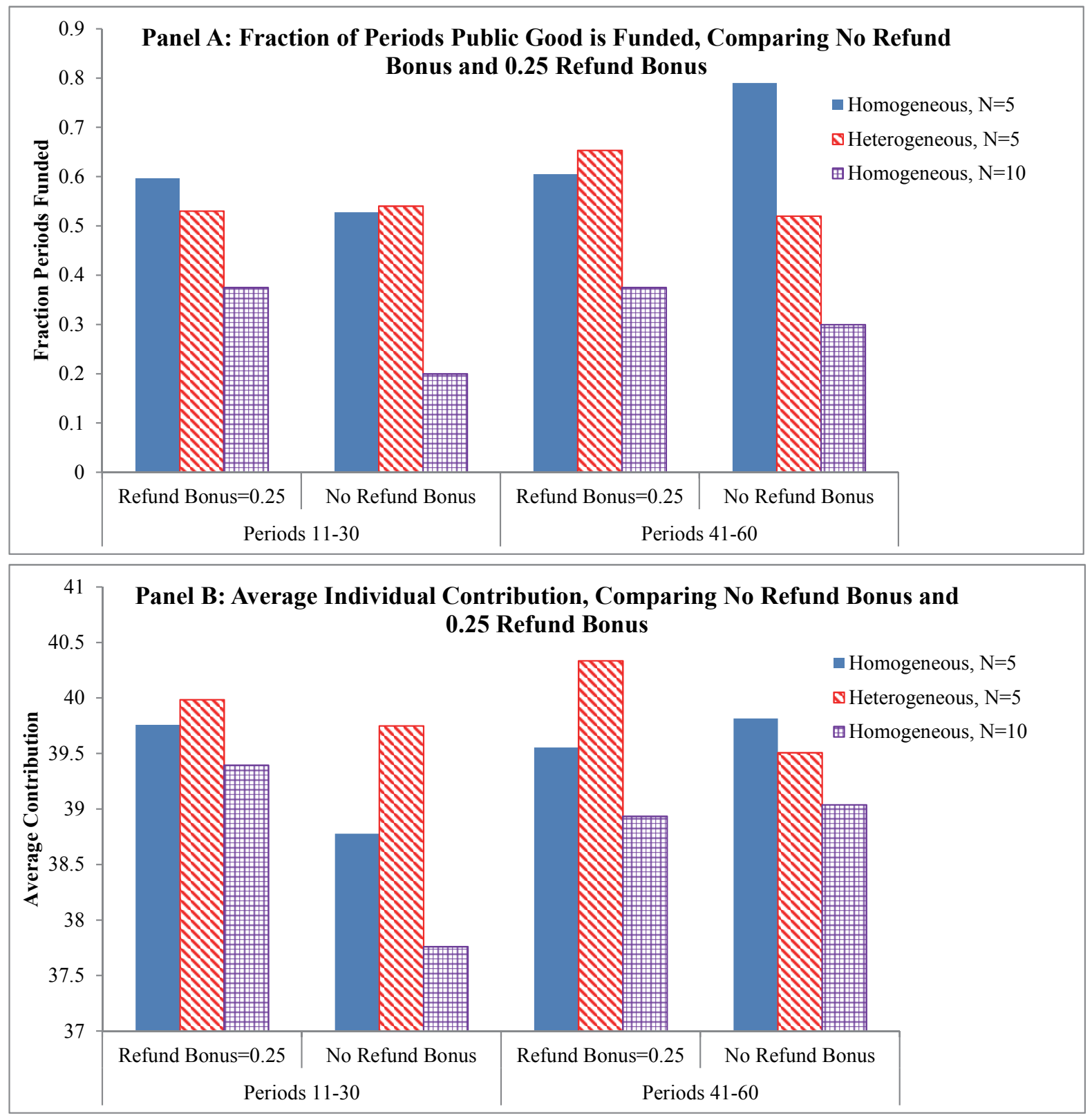

Figure 5: Refund Bonuses vs No Refund Bonuses 
tests used previously to compare the different refund bonus treatments. These tests reveal that the PG provision rate is significantly lower without refund bonuses in the late periods with heterogeneous values $(N=5)$, with large groups $(N=10)$ when pooling across periods, and with large groups in the early periods (for this last result at only the ten-percent significance level). Group contribution levels are significantly lower without refund bonuses in the late periods with heterogeneous values $(N=5)$ and in the early periods with homogeneous values and small groups $(N=5)$. For the large groups $(N=10)$ contributions are lower (at the ten-percent significance level) in the early periods.

\section{Bounded rationality}

While the Nash equilibrium predictions find strong experimental support in the 0.25 and 0.75 refund bonus treatments, support is weaker for the 0.50 treatment. In the latter treatment, the rate of provision is smaller than predicted especially with heterogeneous values and, despite the unique Nash equilibrium, the variance of contributions is no different from other treatments with multiple equilibria. However, if we replace the Nash assumption of perfect rationality with the assumption of bounded rationality that underlies the quantal response equilibrium (QRE), see Rosenthal (1989) and McKelvey and Palfrey (1995), the model obtains a closer fit to experimental data. Refined with bounded rationality, the model can explain subjects' behavior in the 0.50 treatment as well as the observed differences between the homogeneous and heterogeneous value treatments. At the same time, the empirical findings consistent with Nash equilibrium also remain consistent with QRE.

In what follows, we characterize the distribution of aggregate contributions when a consumer's probability of contributing a specific amount increases in the associated expected payoff, and then we discuss the empirical implications. 


\subsection{Distribution of aggregate contributions}

Adopting the continuous version of the logit probabilistic choice framework, we define consumer $i$ 's probability density $f_{i}\left(g_{i}\right)$ of contributing $g_{i}$ by an exponential function

$$
f_{i}\left(g_{i}\right)=\frac{\exp \left(\pi_{i}^{e}\left(g_{i}\right) / \mu\right)}{\int_{0}^{\bar{g}} \exp \left(\pi_{i}^{e}(g) / \mu\right) d g}, \quad i=1, \ldots, N
$$

of the expected payoff $\pi_{i}^{e}$ determined by

$$
\pi_{i}^{e}\left(g_{i}\right)=\left(v_{i}-g_{i}\right) \operatorname{Pr}\left(G \geq C \mid g_{i}\right)+r g_{i} \operatorname{Pr}\left(G<C \mid g_{i}\right) .
$$

In (3), $\mu>0$ is a noise parameter that measures the degree of bounded rationality, and we set an upper bound of $\bar{g}$ on individual contributions. Denoting the distribution of total contributions $G_{-i}$ of consumers other than $i$ by $F_{-i}$, we can express the conditional probabilities of provision and non-provision as $\operatorname{Pr}\left(G \geq C \mid g_{i}\right)=1-F_{-i}\left(C-g_{i}\right)$ and $\operatorname{Pr}\left(G<C \mid g_{i}\right)=F_{-i}\left(C-g_{i}\right)$, respectively. The (logit) QRE is a vector of densities $\left(f_{i}\right)$, $i=1, \ldots, N$, that is a fixed point of $(3)$.

The probability density of aggregate contributions, denoted by $h(G)$, is given by the convolution of individual densities $\left(f_{i}\right)$ equal to

$$
h(G)=\frac{\int_{\mathcal{A}(G)} \exp \left(\sum_{i} \pi_{i}^{e}\left(g_{i}\right) / \mu\right) d \mathbf{g}}{K},
$$

where $\mathcal{A}(G)=\left(\mathrm{g}: \sum_{i} g_{i}=G\right)$ is the set of vectors $\mathbf{g}$ which elements sum up to $G$, and $K$ is a constant that ensures integration to 1 . Thus, the probability density $h(G)$ is proportional to the exponent of the sum of expected individual payoffs when $G$ is contributed in total.

In equilibrium, when the noise parameter $\mu$ is relatively small, the sum of expected individual payoffs conditional on $G$ can be approximated by the sum of actual payoffs

$$
\sum_{i}^{N} \pi_{i}^{e}\left(g_{i}\right) \simeq \sum_{i}^{N} \pi_{i}\left(g_{i}, G\right)=\Pi(G)
$$

The reason is that the probability mass is concentrated around $C$ when $\mu$ is relatively 
small (at the limit $\mu \rightarrow 0$, quantal response equilibrium reduces to Nash equilibrium). It implies that each individual contributor has an accurate account of the behavior of others and, thus, $\operatorname{Pr}\left(G \geq C \mid g_{i}\right)$ can well be approximated by a $0-1$ step function of individual contribution $g_{i}$. Then, the density $h(G)$ is given by

$$
h(G)=\left\{\begin{array}{ccc}
\frac{l(G) \exp (r G / \mu)}{K} & \text { if } \quad G<C, \\
\frac{l(G) \exp ((V-G) / \mu)}{K} & \text { if } \quad G \geq C
\end{array}\right.
$$

where $l(G)$ is the measure of the set $\mathcal{A}(G)$. With little noise, the probability density of $\mathrm{QRE}$ aggregate contributions is proportional to the exponent of the actual aggregate payoff.

When the approximation in (6) is not accurate, a closed-form expression for the density function $h(G)$ arising in QRE is difficult to obtain. In this case, and as it is undertaken in the analysis below, we revert to the numerical solution in determining $h(G)$ using the same parameter values as in the experiment and, in particular, the strategy space of discrete contributions.

\subsection{Empirical implications and evidence}

\section{Aggregate contributions in homogeneous value treatment}

Figure 6 plots the empirical distributions of aggregate contributions in the homogeneous value treatments and Table 4 reports the percentage of periods with contributions that strictly exceed the threshold for all treatments. Comparing the distributions in Figure 6 with the corresponding aggregate payoffs plotted in Figure 1, we can immediately notice considerable similarity between the plots - the graph of the frequency distribution mimics that of the aggregate payoffs. In particular, the sign and the magnitude of the difference between the contribution frequencies around 200 closely match the corresponding differences in aggregate payoffs. In the 0.50 refund bonus treatment, where payoffs differ only slightly when contributions are at and below the provision point, we accordingly observe relatively symmetric frequencies around the provision point. While inconsistent with the Nash equilibrium, the observed pattern of aggregate contributions is consistent 
with QRE predictions made for small levels of noise $\mu$. (Result 3 on coordination patterns shows that consumers manage to coordinate rather closely at the point of provision, providing support for small levels of noise.)

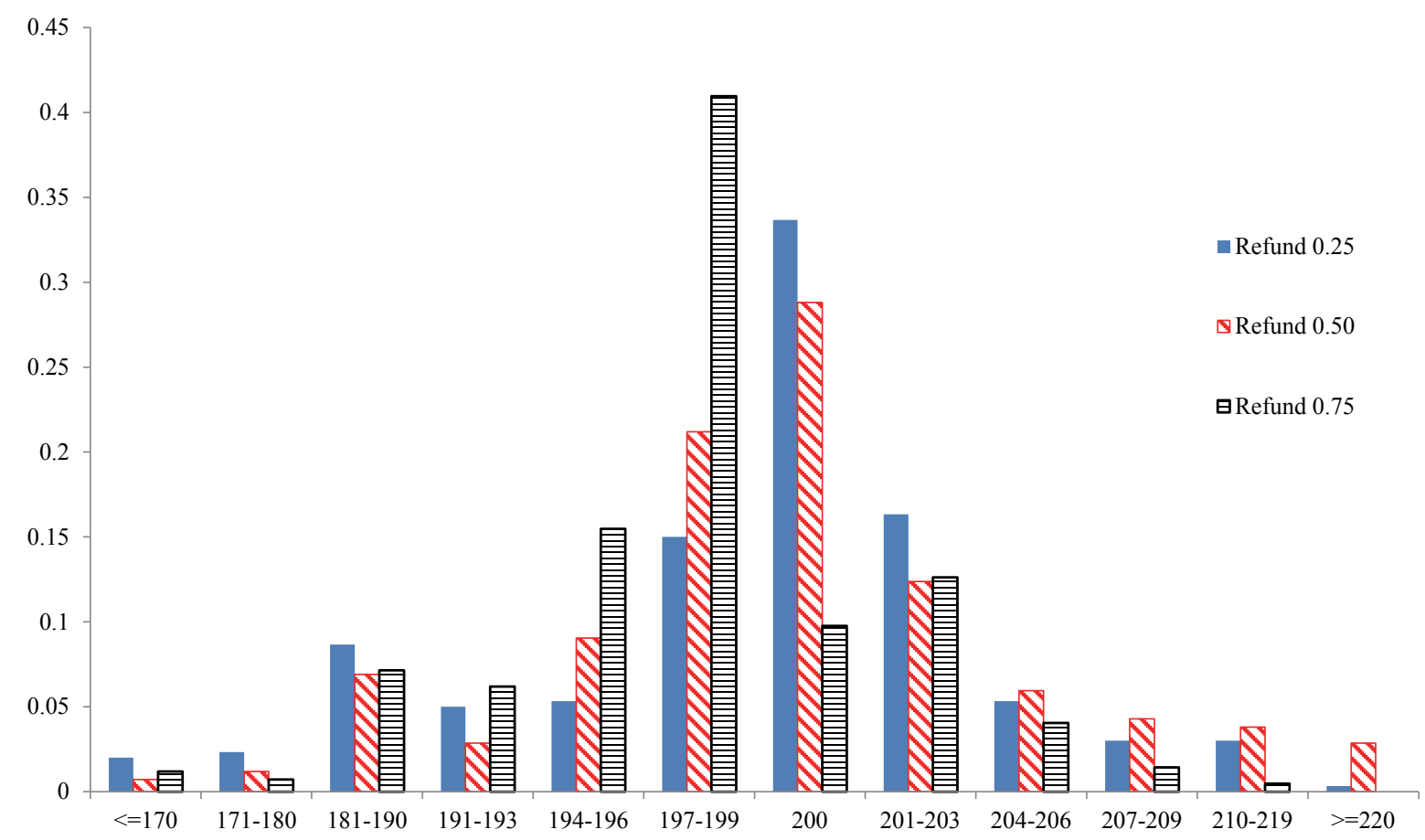

Figure 6: Frequency Distribution of Group Contributions: Homogeneous Periods 31-60

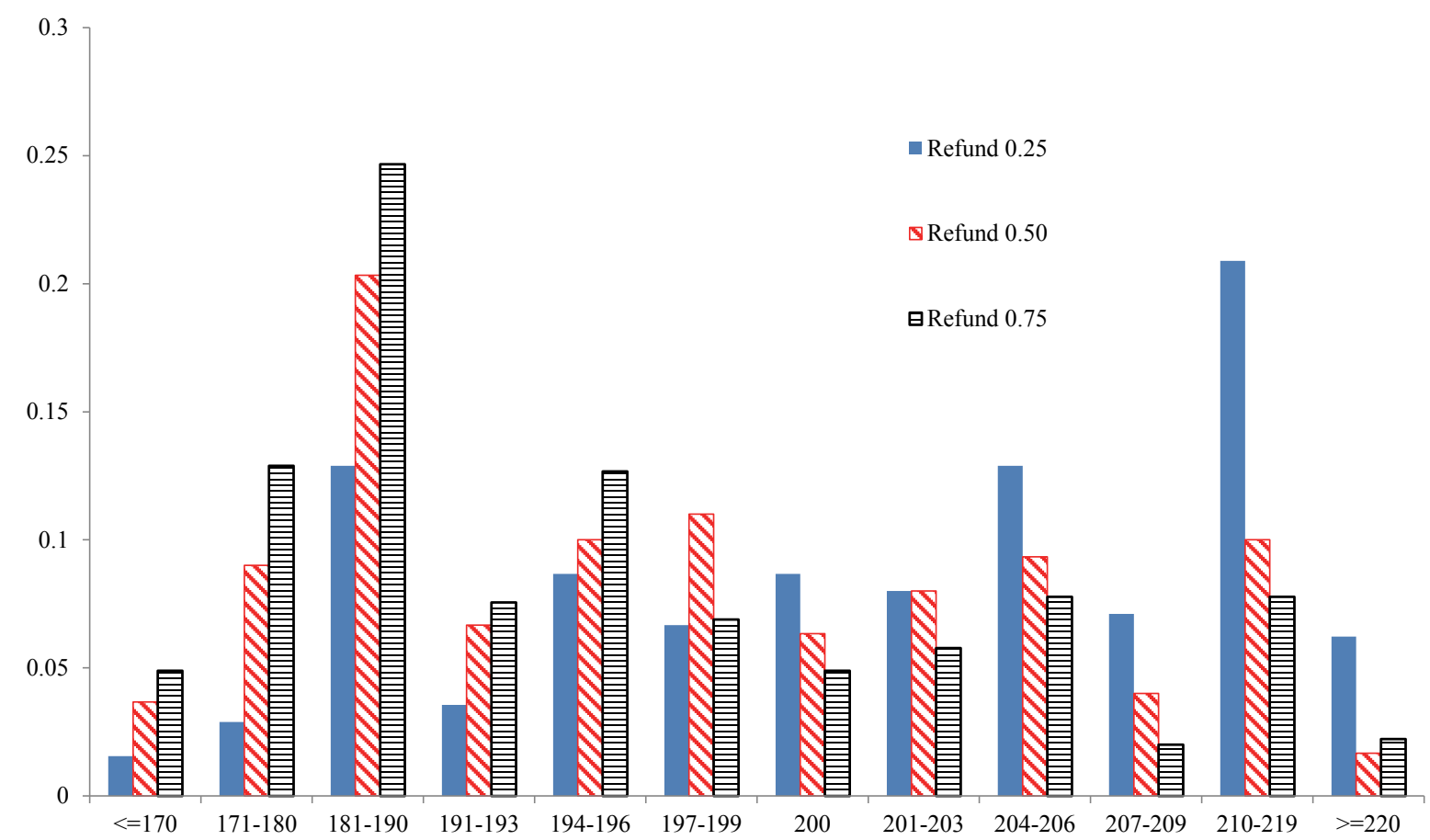

Figure 7: Frequency Distribution of Group Contributions: Heterogeneous Periods 31-60 
Table 4: Percentage of Periods with Excess Contributions, Periods 31-60

\begin{tabular}{lcccc}
\hline & \multicolumn{4}{c}{ Refund Bonus Treatment } \\
& $r=0$ & $r=0.25$ & $r=0.50$ & $r=0.75$ \\
\hline Homogeneous $N=5$ & $37.3 \%$ & $28.0 \%$ & $29.3 \%$ & $18.6 \%$ \\
Heterogeneous $N=5$ & $42.0 \%$ & $55.1 \%$ & $33.0 \%$ & $25.6 \%$ \\
Homogeneous $N=10$ & $31.7 \%$ & $40.0 \%$ & & \\
\hline \hline
\end{tabular}

\section{Homogeneous vs. heterogeneous value treatments}

As Result 4 indicates, there are significant differences in contribution variance between the homogeneous and heterogeneous value treatments. Figure 7 plots the empirical distributions of aggregate contributions in the heterogeneous value treatments, from which we observe a wider dispersion of contributions compared with the homogeneous value case. While not predicted by the Nash equilibrium, this observation, however, is consistent with the predictions of the model of bounded rationality. As the heterogeneous value environment is more complex, we can arguably expect more noise in the decision making process, which implies a higher value of $\mu$ in the model. The consequence of this is a wider dispersion of contributions as differences in payoffs weigh less for probability density as it can be seen from eq. (3).

Comparing Figures 6 and 7, we can notice that the effect of heterogeneity is not uniform across the treatments. In the 0.25 treatment, the effect is a shift of more probability mass to the right from the provision point but to the left in the 0.50 and 0.75 treatments, so producing the differences in the rate of provision and in average aggregate contributions presented in Results 1 and 2, respectively. Table 4 also shows a substantially larger increase with heterogeneity in the instances of excess contributions with $r=0.25$ than with other refund bonus rules. Again, the model of bounded rationality is consistent with this pattern. At the limit $\mu \rightarrow 0$, where the QRE coincides with the Nash equilibrium, the entire probability mass is concentrated around 200 or, respectively, 199 in the 0.75 treatment. An increase in $\mu$ will have an effect on the rate of provision and aggregate contributions depending on the direction of the dispersion of probability mass. When $r$ is small so that $r C<V-C$, more probability mass will spread to the right from 200 
as payoffs are higher there, also implying an increase in expected total contributions. But the converse holds for the case when the refund bonus $r$ is high, $r C \geq V-C$, as more probability mass is attracted to the left of $200 .{ }^{11}$ Intuitively, given noisier behavior, consumers tend to contribute more when the refund bonus is low in order to hedge themselves against the risk of an unfavorable outcome $G<C$, but the converse holds when the refund bonus is high. ${ }^{12}$ Consistent with this intuition, for different levels of noise $\mu$ Figure 8 plots the rates of provision and expected aggregate contributions from the numerical solution of the QRE model iteratively solved for the treatments with homogeneous valuations and the upper bound for individual (discretized) contributions set at $\bar{g}=200$.
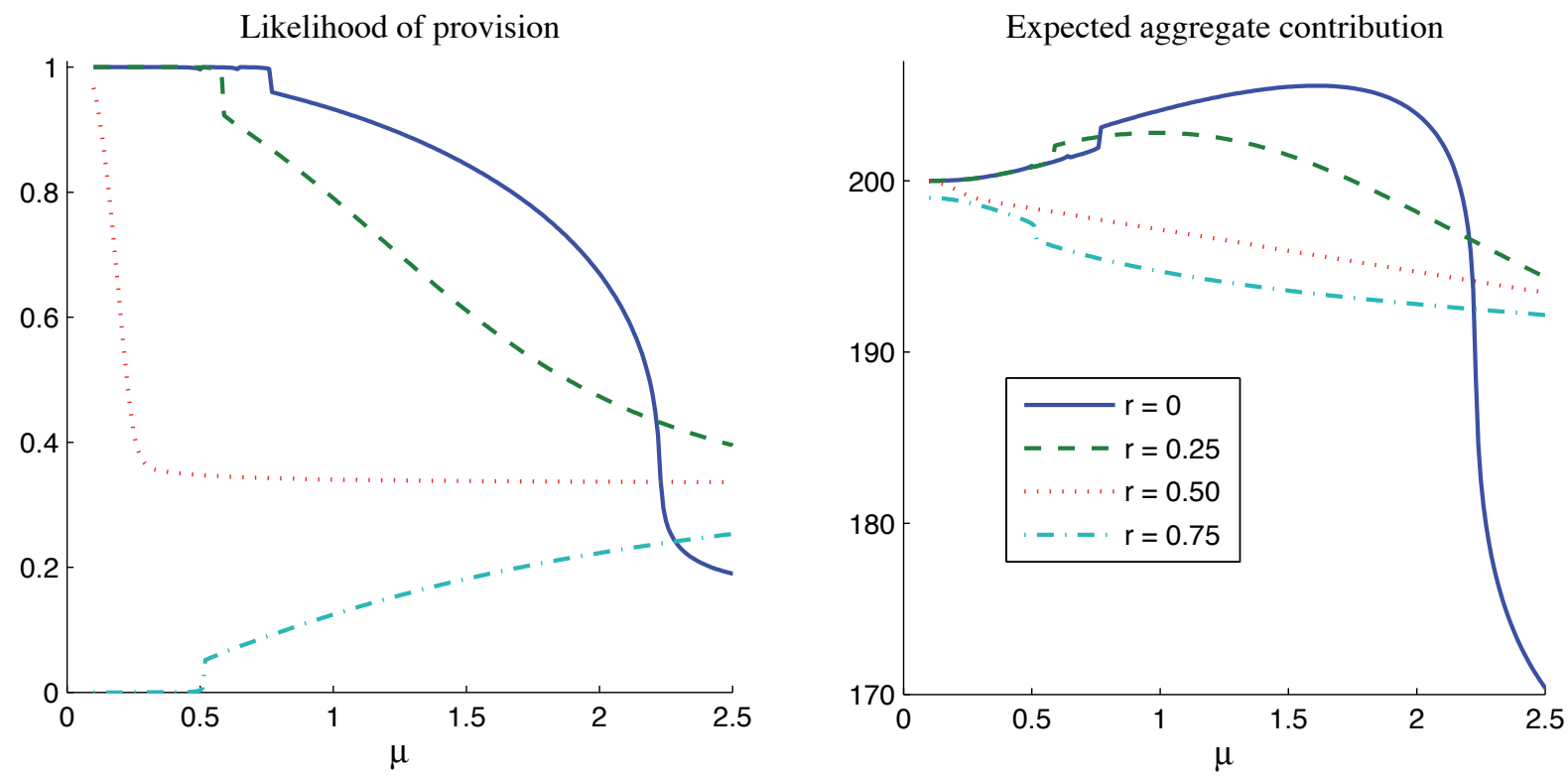

Figure 8: QRE outcomes for different levels of noise when $N=5$

\section{Distributive efficiency}

Result 5 shows that the properties of the mechanism related to distributive efficiency are similar across all refund bonus treatments. From the perspective of Nash equilibrium

\footnotetext{
${ }^{11}$ At $r=0.50$, where $r C=V-C$, the model still predicts more probability mass to the left of 200 as the aggregate payoff decays faster to the right of 200 than to the left.

${ }^{12}$ This intuition is confirmed by comparing individual contributions of consumers who have value $v=60$ in the heterogeneous treatment with contributions in the homogeneous treatment. In the 0.25 heterogeneous value treatment, individual contributions are statistically higher but in the 0.50 and 0.75 treatments they are lower than contributions in the corresponding homogeneous value treatments.
} 
predictions, one could argue that the contribution of about two thirds of the private value is a focal choice in the coordination game irrespective of the size of refund bonus. The model refined with bounded rationality can substantiate this argument. To illustrate this point, Figure 9 plots the QRE individual contribution densities $f_{i}\left(g_{i}\right)$ for the case of heterogenous values with the noise parameter set at $\mu=1 .{ }^{13}$ With refund bonus $r=0.50$, the modal contribution coincides with the Nash equilibrium of two thirds of the private value. For other bonus rules the modal contribution is similar, consistent with the observed similarities in distributive efficiency across the treatments.
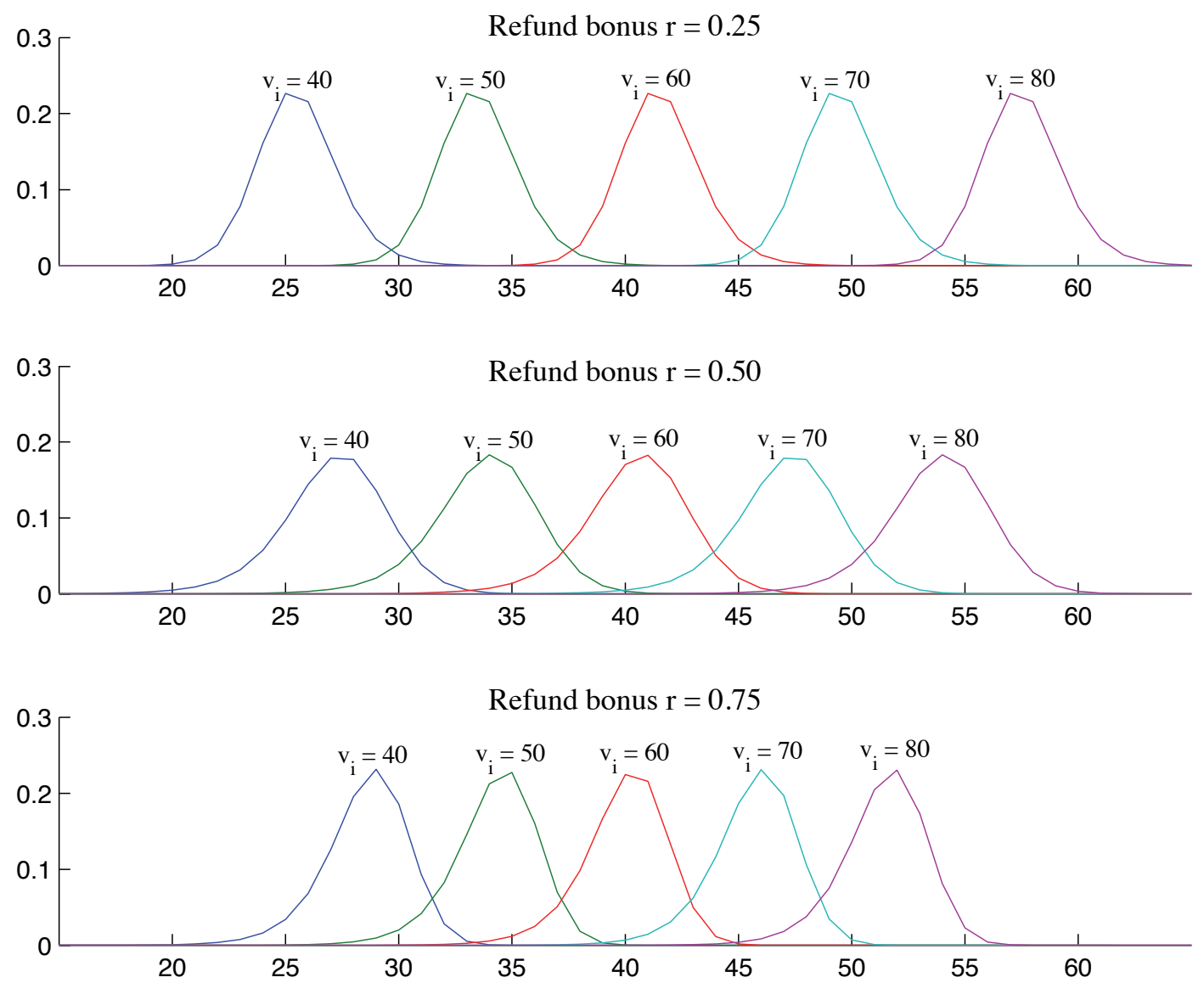

Figure 9: QRE individual contribution densities $f_{i}\left(g_{i}\right)$

\footnotetext{
${ }^{13}$ In calculating QRE for each refund bonus rule, we used the same initial weights uniformly distributed over the individual strategy space set at $\{0,1, \ldots, 199,200\}$. The pattern of individual densities shown in Figure 9 is robust to different initial weights and also to values of noise parameter $\mu$. The choice of $\mu=1$ seems to be in a range appropriate for what we see in the data and numerical QRE solutions presented earlier. In general, the scale for $\mu$ is arbitrary and cannot be compared across studies because it depends directly on the nominal scale for denoting payoffs.
} 


\section{Comparison with no refund bonuses}

Figure 8 also plots the QRE rate of provision and expected aggregate contribution for the standard mechanism of no refund bonuses $(r=0)$. The QRE outcomes conform with the economic intuition presented prior to Hypothesis $6 .{ }^{14}$ In deciding whether and how much to contribute, consumers weigh gains and losses of their decision, where the gain is an increase in the probability of provision and the loss is the monetary cost of contribution. When the level of noise $\mu$ is low, consumers are pivotal with a high probability which prompts them to contribute. Furthermore, as outcomes $G<200$ are less favorable when no refund bonuses are offered, we even see higher contributions compared to the 0.25 refund bonus case. However, with levels of noise increasing, individual consumers have less control over the likelihood of success. Then, as it follows from eq. (4), with the success rate $\operatorname{Pr}\left(G \geq C \mid g_{i}\right)$ becoming less dependent on individual contributions, consumers reduce their contributions because now losses loom larger than gains. As a result, we obtain the rapid decay of the provision rate and expected aggregate contributions depicted in Figure 8. For larger group sizes, this decay starts at lower values of $\mu$ as now an individual contribution becomes less likely to be pivotal, which is illustrated in Figure 10 that compares QRE outcomes for the empirically studied cases of $r=0$ and $r=0.25$ when $N=10$. These QRE patterns are consistent with the empirical evidence presented in Result 6.

\section{Conclusion}

This paper tests a new extension of the provision point mechanism with refund bonuses. We distinguish two motivations for this study, theoretical and practical. Regarding the theoretical motivation, the extension improves the standard provision point mechanism up to strict implementation. In particular, refund bonuses help eliminate inefficient equilibria in the problem of fundraising for public goods and, as a consequence, implement the public good uniquely. The mechanism is robust to information distribution

\footnotetext{
${ }^{14}$ Similarly to the argument of Bagnoli and Lipman (1989) earlier discussed, the zero-contribution outcome is not QRE unless there are nuisance costs of contribution.
} 

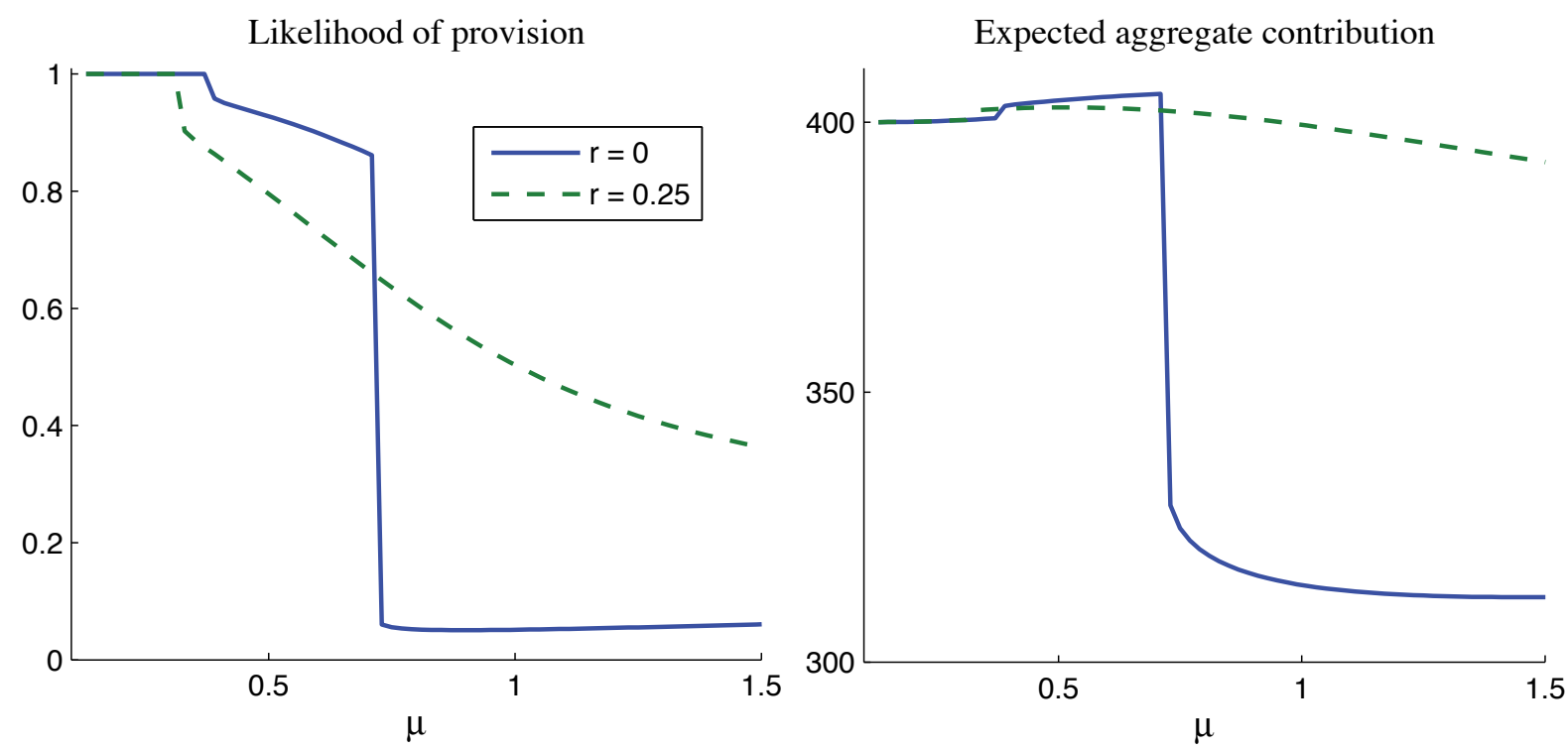

Figure 10: QRE outcomes for different levels of noise when $N=10$ and $C=400$

and can achieve allocative and distributive efficiency. Our experimental results show that subjects respond to the incentives brought about by refund bonuses in predicted ways. Specifically, the Nash equilibrium predictions of allocative and distributive efficiency find strong empirical support, whereas experimental results on equilibrium coordination and invariance to information distribution are better explained by the model refined with bounded rationality.

The practical motivation for this study comes from the potential applicability of the extension given the wide use of the provision point mechanism in crowdfunding. As argued earlier, there are concerns about future growth prospects of crowdfunding and its success rate. This proposed new mechanism may alleviate some of these concerns and this initial empirical evidence is promising, although caution is always warranted when drawing potential policy implications from initial results provided by simplified laboratory experiments. Significantly, our results show that the refund bonus rule need not be generous - the best results are obtained for the least generous refund bonus used, i.e., $r=0.25$. Moreover, the mechanism with a low bonus rate performed equally well in a more complex environment with heterogeneous valuations, and reached a success rate of up to $80 \%$ in later periods. Drawing on the model with bounded rationality supported by our experimental results, a similar performance can be expected even with smaller refund 
bonuses so minimizing the risks involved, but this question is left for future research.

There are more avenues for future research that should be explored to investigate the practical applicability of the mechanism with refund bonuses. An important question is how the mechanism performs in a dynamic setting akin to that used by crowdfunding platforms. A dynamic setting may further alleviate the problem of equilibrium coordination, which, as our results show, is still present. Another interesting question relates to the source of refund bonuses and its effect on contribution behavior. In our experiment, the source of refund bonuses is exogenous, yet in practical applications it would need to be endogenous, e.g., a campaigner's own contribution, first-movers' seed money, or insurance premiums paid by successful campaigners. Lastly, more research is needed on aggregate uncertainty and group size effects, which could be ideally done in a field study.

\section{Acknowledgments}

We would like to thank Pavlo Blavatskyy, two anonymous referees, and numerous seminar and conference audiences for valuable suggestions. The Swiss National Science Foundation provided financial support (Project 100018_150138).

\section{References}

Bagnoli, M., Lipman, B. L., 1989. Provision of Public Goods: Fully Implementing the Core through Private Contributions. Rev. Econ. Stud. 56, 583-601.

Bagnoli, M., McKee, M., 1991. Voluntary Contributions Games: Efficient Private Provision of Public Goods. Econ. Inquiry 29, 351-366.

Castillo, M., Petrie, R., Wardell, C., 2014. Fundraising through online social networks: A field experiment on peer-to-peer solicitation. J. Public Econ. 114, 29-35.

Chen, Y., 2008. Incentive-Compatible Mechanisms for Pure Public Goods: A Survey of Experimental Research. In: Plott, C.R., \& Smith, V.L. (eds), Handbook of Experimental Economics Results, vol. 1. Elsevier, North-Holland, pp. 625-643.

Clarke, E.H., 1971. Multipart Pricing of Public Goods. Public Choice 11, 17-33. 
Corazzini, L., Faravelli, M., Stanca, L., 2010. A Prize To Give For: An Experiment on Public Good Funding Mechanisms. Econ. J. 120, 944-967.

Croson, R. T. A., Marks, M.B., 2000. Step Returns in Threshold Public Goods: A Metaand Experimental Analysis. Exper. Econ. 2, 239-259.

Falkinger, J., 1996. Efficient private provision of public goods by rewarding deviations from average. J. Public Econ. 62, 413-422.

Falkinger, J., Fehr, E., Gaechter, S., Winter-Ebmer, R., 2000. A Simple Mechanism for the Efficient Provision of Public Goods: Experimental Evidence. Amer. Econ. Rev. 90, $247-264$.

Fischbacher, U., 2007. z-Tree: Zurich toolbox for ready-made economic experiments. Exper. Econ. 10, 1386-1457.

Gerber, A., Wichardt, P.C., 2009. Providing public goods in the absence of strong institutions. J. Public Econ. 93, 429-439.

Gerber, A., Neitzel, J., Wichardt, P.C., 2013. Minimum Participation Rules for the Provision of Public Goods. Europ. Econ. Rev. 64, 209-222.

Goeree, J.K., Maasland, E., Onderstal, S., Turner, J.L., 2005. How (Not) to Raise Money. J. Polit. Economy. 113, 897-918.

Greiner, B., 2004. An Online Recruitment System for Economic Experiments. In: K. Kremer, V. Macho (Eds.), Forschung und Wissenschaftliches Rechnen GWDG Bericht 63, Gesellschaft fr Wissenschaftliche Datenverarbeitung, Gttingen, pp. 79-93.

Groves, T., 1973. Incentives in Teams. Econometrica 41, 617-631.

Groves, T., Ledyard, J., 1977. Optimal Allocation of Public Goods: A Solution to the "Free Rider" Problem. Econometrica 45, 783-809.

Kearney, M. S., 2005. State lotteries and consumer behavior. J. Public Econ. 89, 22692299. 
Kickstarter, 2015. Kickstarter stats, https://www.kickstarter.com/help/stats, retrieved on 12 Feb 2015.

Kominers, S. D., Weyl, E. G., 2012. Holdout in the Assembly of Complements: A Problem for Market Design. Amer. Econ. Rev. 102, 360-365.

Kuppuswamy, V., Bayus, B. L., 2014. Crowdfunding Creative Ideas: The Dynamics of Project Backers in Kickstarter, University of North Carolina, Kenan-Flagler Research Paper No. 2013-15.

Lange, A., List, J.A., Price, M.K., 2007. Using Lotteries to Finance Public Goods: Theory and Experimental Evidence. Int. Econ. Rev. 48, 901-927.

Ledyard, J., 1995. Public goods: A survey of experimental research. In: Kagel, J., \& Roth, A. (eds), The handbook of experimental economics. Princeton: Princeton University Press, pp. 111-194.

Mailath, G.J., Postlewaite, A., 1990. Asymmetric Information Bargaining Problems with Many Agents. Rev. Econ. Stud. 57, 351-367.

Marks, M., Croson, R., 1999. The effect of incomplete information in a threshold public goods experiment. Public Choice 99, 103-118.

Massolution, 2014. 2013CF Crowdfunding Industry Report, http://crowdsourcing.org/l/20898.

Masuda, T., Okano, Y., Saijo, T., 2014. The minimum approval mechanism implements the efficient public good allocation theoretically and experimentally. Games Econ. Behav. 83, 73-85.

McKelvey, R.D., Palfrey, T.R., 1995. Quantal response equilibria for normal form games. Games Econ. Behav 10, 6-38.

Morgan, J., 2000. Financing Public Goods by Means of Lotteries. Rev. Econ. Stud. 67, $761-784$. 
Morgan, J., Sefton, M., 2000. Funding Public Goods with Lotteries: Experimental Evidence. Rev. Econ. Stud. 67, 785-810.

Olson, M., 1965. The Logic of Collective Action: Public Goods and the Theory of Groups. Cambridge, MA: Harvard University Press.

Palfrey, T.R., Rosenthal, H., 1984. Participation and the provision of discrete public goods: a strategic analysis. J. Public Econ. 24, 171-193.

Reuben, E., Riedl, A., 2013. Enforcement of Contribution Norms in Public Good Games with Heterogeneous Populations. Games Econ. Behav. 77, 122-137.

Rosenthal, R.W., 1989. A bounded rationality approach to the study of noncooperative games. Int. J. Game Theory 18, 273-292.

Tabarrok, A., 1998. The private provision of public goods via dominant assurance contracts. Public Choice 96, 345-362.

The Verge, 2013. Indie no-go: only one in ten projects gets fully funded on Kickstarter's biggest rival, August 7, 2013, http://www.theverge.com/2013/8/7/4594824/less-than10-percent-of-projects-on-indiegogo-get-fully-funded

Varian, H.R., 1994. A Solution to the Problem of Externalities When Agents Are WellInformed. Amer. Econ. Rev. 84, 1278-1293.

Zubrickas, R., 2013. The provision point mechanism with reward money. University of Zurich, Department of Economics, Working Paper \#114.

Zubrickas, R., 2014. The provision point mechanism with refund bonuses. J. Public Econ. $120,231-234$. 